\title{
A Unified Model of Phantom Energy and Dark Matter ${ }^{\star}$
}

Max CHAVES ${ }^{\dagger}$ and Douglas SINGLETON $\ddagger$

${ }^{\dagger}$ Escuela de Fisica Universidad de Costa Rica, San Jose, Costa Rica

E-mail: mchaves@cariari.ucr.ac.cr

$\ddagger$ Physics Department, CSU Fresno, Fresno, CA 93740-8031, USA

E-mail: dougs@csufresno.edu

Received November 01, 2007, in final form January 22, 2008; Published online January 30, 2008

Original article is available at http://www.emis.de/journals/SIGMA/2008/009/

\begin{abstract}
To explain the acceleration of the cosmological expansion researchers have considered an unusual form of mass-energy generically called dark energy. Dark energy has a ratio of pressure over mass density which obeys $w=p / \rho<-1 / 3$. This form of massenergy leads to accelerated expansion. An extreme form of dark energy, called phantom energy, has been proposed which has $w=p / \rho<-1$. This possibility is favored by the observational data. The simplest model for phantom energy involves the introduction of a scalar field with a negative kinetic energy term. Here we show that theories based on graded Lie algebras naturally have such a negative kinetic energy and thus give a model for phantom energy in a less ad hoc manner. We find that the model also contains ordinary scalar fields and anti-commuting (Grassmann) vector fields which act as a form of two component dark matter. Thus from a gauge theory based on a graded algebra we naturally obtained both phantom energy and dark matter.
\end{abstract}

Key words: dark energy; phantom energy; graded algebras

2000 Mathematics Subject Classification: 81R10; 81T10

\section{Introduction}

Graded Lie algebras or Lie superalgebras (i.e. algebras having commuting and anti-commuting generators) were at one time considered as models for a more complete unified electroweak theory [1] as well as Grand Unified Theories [2]. Such graded algebras had many attractive features: vector and scalar bosons were contained within the same theory, the Weinberg angle was fixed; in some formulations the mass of the Higgs was fixed. However these graded algebras generically [3] gave rise to negative kinetic energy terms for some of the gauge fields when the graded trace or supertrace was used.

Here we show that this negative kinetic energy of the original graded algebras can be used to construct a model for phantom energy $[4,5]$. In addition to the phantom field there are other fields which come from this model which act as dark matter. The advantage of the combined phantom energy/dark matter model presented here is that it is derived from a modified gauge principle (i.e. the gauge principle applied to graded algebras) rather than being introduced by hand. This feature fixes the parameters, such as the coupling between the phantom energy and dark matter, that are free in more phenomenological models.

Phantom energy is a form of dark energy which has a ratio of pressure over density given by $w=p / \rho<-1$. Dark energy in general is a cosmological "fluid" with $w<-1 / 3$, which gives rise

\footnotetext{
${ }^{\star}$ This paper is a contribution to the Proceedings of the Seventh International Conference "Symmetry in Nonlinear Mathematical Physics" (June 24-30, 2007, Kyiv, Ukraine). The full collection is available at http://www.emis.de/journals/SIGMA/symmetry2007.html
} 
to an accelerated cosmological expansion. Dark energy was proposed to explain the accelerated expansion observed in studies of distant type Ia supernova [6, 7]. There are various proposals as to the nature of dark energy: a small, positive cosmological constant, quintessence [8], brane world models [9, 10], Chaplyin gas [11], $k$-essense [12], axionic tensor fields [13] and others. A good review can be found in [14]. Phantom energy is an extreme form of dark energy. The simplest model for phantom energy involves a scalar field with a negative kinetic energy term [4]

$$
\mathcal{L}_{p}=-\frac{1}{2}\left(\partial_{\mu} \phi\right)\left(\partial^{\mu} \phi\right)-V(\phi) .
$$

The negative sign in front of the kinetic energy term makes this an unusual field theory. Theories with negative kinetic energies have been investigated theoretically starting with [15]. Other papers considering scalar fields with negative kinetic energies can be found in [16]. The main objection to these negative kinetic energy theories is that quantum mechanically they violate either conservation of probability or they have no stable vacuum state due to an unbounded, negative energy density. Although such unusual field theories are not ruled out [4], one can place constraints on them [17]. Despite the theoretical problems of a scalar field with a negative kinetic energy term the reason to consider such a strange field theory is that recent observations give $-1.48<w<-0.72[18]$ and thus favor $w<-1$. A recent comparison of data from various sources can be found in [19].

The result $w<-1$ coming from the Lagrangian in (1) depends not only on the negative kinetic energy term, but also requires that the potential, $V(\phi)$, be present and satisfy some conditions. One can calculate $p$ and $\rho$ from (1). Assuming that the scalar field is spatially homogeneous enough so that only the time variation is important one finds

$$
w=\frac{p}{\rho}=\frac{-\frac{1}{2} \dot{\phi}^{2}-V(\phi)}{-\frac{1}{2} \dot{\phi}^{2}+V(\phi)} .
$$

In order to have $w<-1$ the potential must satisfy $\sqrt{2 V(\phi)}>|\dot{\phi}| \geq 0$. We will show that it is possible, using graded algebras, to construct a field theory that satisfies these conditions and so gives rises to phantom energy. Unlike other models, the negative kinetic term comes from the structure of the graded algebras rather than being put in by hand. In addition there are other fields which play the role of dark matter.

\section{Review of $S U(2 / 1)$ algebra}

Here we briefly review the graded algebra $S U(2 / 1)$. The basic idea of using graded algebras to give phantom energy works for larger graded algebras like $S U(N / 1)$ with $N>2$. We have taken $S U(2 / 1)$ for simplicity.

We use the representation for $S U(2 / 1)$ which consists of the following eight $3 \times 3$ matrices

$$
\begin{array}{rlrl}
\text { even: } & T_{1}=\frac{1}{2}\left(\begin{array}{ccc}
0 & 1 & 0 \\
1 & 0 & 0 \\
0 & 0 & 0
\end{array}\right), & T_{2}=\frac{1}{2}\left(\begin{array}{ccc}
0 & -i & 0 \\
i & 0 & 0 \\
0 & 0 & 0
\end{array}\right), \\
T_{3}=\frac{1}{2}\left(\begin{array}{ccc}
1 & 0 & 0 \\
0 & -1 & 0 \\
0 & 0 & 0
\end{array}\right), & T_{8}=\frac{1}{2}\left(\begin{array}{ccc}
1 & 0 & 0 \\
0 & 1 & 0 \\
0 & 0 & 2
\end{array}\right), \\
\text { odd: } & T_{4}=\frac{1}{2}\left(\begin{array}{ccc}
0 & 0 & 1 \\
0 & 0 & 0 \\
1 & 0 & 0
\end{array}\right), & T_{5}=\frac{1}{2}\left(\begin{array}{ccc}
0 & 0 & -i \\
0 & 0 & 0 \\
i & 0 & 0
\end{array}\right),
\end{array}
$$




$$
T_{6}=\frac{1}{2}\left(\begin{array}{ccc}
0 & 0 & 0 \\
0 & 0 & 1 \\
0 & 1 & 0
\end{array}\right), \quad T_{7}=\frac{1}{2}\left(\begin{array}{ccc}
0 & 0 & 0 \\
0 & 0 & -i \\
0 & i & 0
\end{array}\right)
$$

Except for $T_{8}$ this is the standard, fundamental representation of $S U(3)$. The matrices on the first line above (i.e. $T_{1}, T_{2}, T_{3}, T_{8}$ ) are the even generators, and those on the second line (i.e. $T_{4}$, $\left.T_{5}, T_{6}, T_{7}\right)$ are odd generators. The even generators satisfy commutation relationships among themselves which can be written symbolically as [even, even] = even. Mixtures of even and odd generators satisfy commutators of the form [even, odd] = odd. Finally the odd generators satisfy anti-commutation relationships of the form $\{$ odd, odd $\}=$ even. The further details of the $S U(2 / 1)$ graded algebra can be found in the paper by Dondi and Jarvis [1] or in Ecclestone [3]. The odd generators above are different than those usually taken in the literature. The connection of the odd generators above with those in [1] is given by $\bar{Q}^{1}, Q_{1}=T_{4} \pm i T_{5}$ and $\bar{Q}^{2}, Q_{2}=T_{6} \pm i T_{7}$. In the rest of the article we will use the convention that generators with indices from the middle of the alphabet $(i, j, k)$ are the even generators, $T_{1}, T_{2}, T_{3}, T_{8}$, while indices from the beginning of the alphabet $(a, b, c)$ are the odd generators $T_{4}, T_{5}, T_{6}, T_{7}$.

For the graded algebra one replaces the concept of the trace by the supertrace. For $S U(2 / 1)$ this means that one writes some general element of the group as

$$
M=\left(\begin{array}{cc}
A_{2 \times 2} & B_{2 \times 1} \\
C_{1 \times 2} & d_{1 \times 1}
\end{array}\right) .
$$

The subscripts indicate the size of the sub-matrix. The supertrace is now defined as

$$
\operatorname{str}(M)=\operatorname{tr}[A]-\operatorname{tr}[d]
$$

which differs from the regular trace due to the minus sign in front of $d$.

Later we will need the supertraces of the various products of the eight generators $\left(T_{i}, T_{a}\right)$. We collect these results here. For products of even generators we have

$$
\operatorname{str}\left(T_{i} T_{j}\right)=\delta_{i j} \frac{1}{2} \quad \text { except } \quad \operatorname{str}\left(T_{8} T_{8}\right)=-\frac{1}{2}
$$

for the odd generators we have

$$
\operatorname{str}\left(T_{4} T_{5}\right)=-\operatorname{str}\left(T_{5} T_{4}\right)=\frac{i}{2}, \quad \operatorname{str}\left(T_{6} T_{7}\right)=-\operatorname{str}\left(T_{7} T_{6}\right)=\frac{i}{2} .
$$

All other supertraces of the product of two matrices are zero.

\section{Phantom energy and dark matter from an $S U(2 / 1)$ graded algebra}

In [1] vector fields were associated with the even generators and scalar fields with the odd generators as

$$
A_{\mu}=i g A_{\mu}^{i} T_{i}^{\mathrm{even}}, \quad \phi=-g \varphi^{a} T_{a}^{\mathrm{odd}} .
$$

The fields $A_{\mu}^{i}$ are regular commuting fields while $\varphi^{a}$ are Grassmann fields. In block form one can write (6) as

$$
A_{M}=\left(\begin{array}{ccc}
A_{\mu}^{3}+A_{\mu}^{8} & A_{\mu}^{1}-i A_{\mu}^{2} & \varphi^{4}-i \varphi^{5} \\
A_{\mu}^{1}+i A_{\mu}^{2} & -A_{\mu}^{3}+A_{\mu}^{8} & \varphi^{6}-i \varphi^{7} \\
\varphi^{4}+i \varphi^{5} & \varphi^{6}+i \varphi^{7} & 2 A_{\mu}^{8}
\end{array}\right) .
$$


In this fashion, and by using the regular trace, Dondi and Jarvis [1] showed that the Lagrangian

$$
\mathcal{L}=\frac{1}{2 g^{2}} \operatorname{tr}\left(F_{M N} F^{M N}\right), \quad F_{M N}=\partial_{M} A_{N}-\partial_{N} A_{M}+\left[A_{M}, A_{N}\right],
$$

reduced to an $S U(2) \times U(1)$ Yang-Mills Lagrangian for $A_{\mu}$ and a Higgs-like Lagrangian for $\phi$. In (7) we use a different overall sign for the Lagrangian as compared to [1]. This comes because we have chosen different factors of $i$ in the vector potentials defined below in (8). Using such an $S U(2 / 1)$ algebra gave a more unified electroweak theory. The theory based on the graded $S U(2 / 1)$ algebra was more unified in two ways: (i) There was only one coupling constant $g$ rather than two separate coupling constant in the usual Standard Model based on $S U(2) \times U(1)$. Thus in the Standard Model based on the graded $S U(2 / 1)$ algebra the Weinberg angle was predicted rather than being an input parameter. (ii) Second the theory based on the graded $S U(2 / 1)$ algebra automatically had a scalar field coming from the odd terms in (6).

However on further investigation [3] there were problems with using of the graded $S U(2 / 1)$ algebra to construct an electroweak theory. If in (7) one used the correct $S U(2 / 1)$ invariant supertrace then the Yang-Mills part of the reduced Lagrangian would have the wrong sign for the kinetic term for the $U(1)$ gauge field and the kinetic energy term for the scalar field would be lost.

Here we use these apparent negative features to construct a model for phantom energy. Instead of making the association between even/odd generators and vector/scalar fields made in (6) we take the opposite choice [20]

$$
A_{\mu}=i g A_{\mu}^{a} T_{a}^{\text {odd }}, \quad \phi=-g \varphi^{i} T_{i}^{\text {even }} .
$$

Because of the reversal of roles relative to (6) the fields $A_{\mu}^{a}$ are Grassmann fields while $\varphi^{i}$ are regular, commuting fields. Then taking the correct, $S U(2 / 1)$ invariant, supertrace we find that one of the scalar fields develops a negative kinetic energy term in addition to having a potential term which is positive definite. Thus the graded algebra gives rise to a phantom field.

With the choice in (8) the Lagrangian in (7) reduces as follows [20]

$$
\mathcal{L}=\frac{1}{2 g^{2}} \operatorname{str}\left(F_{M N} F^{M N}\right)=\frac{1}{2 g^{2}} \operatorname{str}\left[\left(\partial_{[\mu} A_{\nu]}+\left[A_{\mu}, A_{\nu}\right]\right)^{2}\right]+\frac{1}{g^{2}} \operatorname{str}\left[\left(\partial_{\mu} \phi+\left[A_{\mu}, \phi\right]\right)^{2}\right] .
$$

We have introduced the notation $\partial_{[\mu} A_{\nu]}=\partial_{\mu} A_{\nu}-\partial_{\nu} A_{\mu}$. Note that in comparison to other works such as [1] and [3] we have not introduced extra Grassmann coordinates, $\zeta^{\alpha}$ in addition to the normal Minkowski coordinates $x^{\mu}$. Thus in [1] and [3] coordinates and indices ran over six values - four Minkowski and two Grassmann. The final result in (9) can be obtained from [1] by dropping the Grassmann coordinates.

We first focus on the scalar term in (9). Inserting $\phi$ and $A_{\mu}$ from (8) into the last term in (9) we find [20]

$$
\begin{aligned}
\mathcal{L}_{S} & =\frac{1}{g^{2}} \operatorname{str}\left[\left(\partial_{\mu} \phi+\left[A_{\mu}, \phi\right]\right)^{2}\right] \\
& =\operatorname{str}\left[\left(\partial_{\mu} \varphi^{8} T_{8}+i g A_{\mu}^{a} \varphi^{8}\left[T_{a}, T_{8}\right]\right)^{2}\right]+\operatorname{str}\left[\left(\partial_{\mu} \varphi^{i} T_{i}+i g A_{\mu}^{a} \varphi^{i}\left[T_{a}, T_{i}\right]\right)^{2}\right] .
\end{aligned}
$$

The first term in (10) takes the form of a phantom energy field. Expanding the first term in (10) gives

$$
\mathcal{L}_{\text {Phantom }}=\operatorname{str}\left[\left(\partial_{\mu} \varphi^{8} T_{8}-g A_{\mu}^{4} \varphi^{8} T_{5} / 2+g A_{\mu}^{5} \varphi^{8} T_{4} / 2-g A_{\mu}^{6} \varphi^{8} T_{7} / 2+g A_{\mu}^{7} \varphi^{8} T_{6} / 2\right)^{2}\right] .
$$

We have used the representation of the $S U(2 / 1)$ matrices from the previous section to evaluate the commutators. Using the supertrace results from (4) and (5) the expression in (11) yields

$$
\mathcal{L}_{\text {Phantom }}=-\frac{1}{2}\left(\partial_{\mu} \varphi^{8}\right)^{2}-\frac{1}{16} g^{2}\left(\varphi^{8}\right)^{2}\left(A_{\mu}^{+} A^{-\mu}-A_{\mu}^{-} A^{+\mu}+B_{\mu}^{+} B^{-\mu}-B_{\mu}^{-} B^{+\mu}\right)
$$


with $A_{\mu}^{ \pm}=A_{\mu}^{4} \pm i A_{\mu}^{5}$ and $B_{\mu}^{ \pm}=A_{\mu}^{6} \pm i A_{\mu}^{7}$. Both $A_{\mu}^{ \pm}$and $B_{\mu}^{ \pm}$are Grassmann so the last line in (12) can be written

$$
\mathcal{L}_{\text {Phantom }}=-\frac{1}{2}\left(\partial_{\mu} \varphi^{8}\right)^{2}-\frac{1}{8} g^{2}\left(\varphi^{8}\right)^{2}\left(A_{\mu}^{+} A^{-\mu}+B_{\mu}^{+} B^{-\mu}\right) .
$$

This is of the form of the phantom energy Lagrangian in (1) but with the potential involving not only the scalar field, $\varphi^{8}$, but Grassmann vector fields, $A_{\mu}^{ \pm}$and $B_{\mu}^{ \pm}$. We will discuss these shortly. The minus sign in front of the kinetic energy term comes from taking the $S U(2 / 1)$ invariant supertrace rather than the ordinary trace (see the second supertrace result in (4)).

We next focus on the other scalar fields, $\varphi^{i}, i=1,2,3$ which come from the second term in (10). The calculation proceeds as in equations (11), (12) but with $\varphi^{8}$ replaced by $\varphi^{i}, i=1,2,3$. For example for $\varphi^{1}(11)$ becomes

$$
\mathcal{L}_{\varphi^{1}}=\operatorname{str}\left[\left(\partial_{\mu} \varphi^{1} T_{1}+g A_{\mu}^{4} \varphi^{1} T_{7} / 2-g A_{\mu}^{5} \varphi^{1} T_{6} / 2-g A_{\mu}^{6} \varphi^{1} T_{5} / 2+g A_{\mu}^{7} \varphi^{1} T_{4} / 2\right)^{2}\right]
$$

and (12) becomes

$$
\mathcal{L}_{\varphi^{1}}=\frac{1}{2}\left(\partial_{\mu} \varphi^{1}\right)^{2}-\frac{1}{8} g^{2}\left(\varphi^{1}\right)^{2}\left(A_{\mu}^{+} A^{-\mu}+B_{\mu}^{+} B^{-\mu}\right) .
$$

There are two keys points: the kinetic term for $\varphi^{1}$ is positive $\operatorname{since} \operatorname{str}\left(T_{1} T_{1}\right)=+1 / 2$, and the potential term is the same as for $\varphi^{8}$. The other two even scalar fields follow the same pattern so that in total one can write

$$
\mathcal{L}_{D M}=\frac{1}{2}\left(\partial_{\mu} \varphi^{i}\right)^{2}-\frac{1}{8} g^{2}\left(\varphi^{i}\right)^{2}\left(A_{\mu}^{+} A^{-\mu}+B_{\mu}^{+} B^{-\mu}\right)
$$

where $i$ is summed from 1 to 3 . Thus the total scalar field Lagrangian resulting from (10) is the sum of (13) and (16). The scalar field in (13) has the "wrong" sign for the kinetic term and acts as a phantom field. The scalar fields in (16) are ordinary scalar field which we will interpret as a dark matter candidate. The phantom field and dark matter fields are coupled through the $A_{\mu}^{ \pm}$ and $B_{\mu}^{ \pm}$fields. Thus our model provides a coupling between phantom energy and dark matter. Other models have been considered [21] where there is coupling between dark/phantom energy and dark matter.

We will now examine the Grassmann vector fields, $A_{\mu}^{4}, A_{\mu}^{5}, A_{\mu}^{6}, A_{\mu}^{7}$. The final Lagrangian for these fields will have a nonlinear interaction between the $A_{\mu}^{ \pm}$and $B_{\mu}^{ \pm}$fields. In analogy with QCD we argue that these fields form permanently confined condensates like $\left\langle A_{\mu}^{4} A_{\mu}^{5}\right\rangle$ or $\left\langle A_{\mu}^{+} A_{\mu}^{-}\right\rangle$. These then supply potential (mass-like) terms for the phantom energy and scalar fields of (13) and (16). This also avoids violation of the spin-statistics theorem since these condensates have bosonic statistics (they are composed of two Grassmann fields) and integer spin (they are composed of two integer spin fields). Having a potential term is crucial for the interpretation of $\varphi^{8}$ as a phantom energy field, since for a massless, non-interacting scalar field reversing the sign of the kinetic energy term does not lead a phantom field with $w<-1$ as can be seen from (2) if $V(\phi)=0$. From (9) the vector part of the Lagrangian can be expanded as

$$
\mathcal{L}_{V}=-\frac{1}{2} \operatorname{str}\left[\left(\partial_{[\mu} A_{\nu]}^{a} T_{a}\right)^{2}\right]+\frac{g^{2}}{2} \operatorname{str}\left[\left(A_{\mu}^{a} A_{\nu}^{b}\left\{T_{a}, T_{b}\right\}\right)^{2}\right]=\mathcal{L}_{V 1}+\mathcal{L}_{V 2} .
$$

The commutator has become an anticommutator due to the Grassmann nature of the $A_{\mu}^{a}$ 's. Also note that there is no cubic cross term between the derivative and anticommutator part. This comes about since the anticommutator, $\left\{T_{a}, T_{b}\right\}$ results in even generators, and the supertrace between odd and even generators vanishes. $\mathcal{L}_{V 1}$ is a kinetic term for the fields and $\mathcal{L}_{V 2}$ a potential term. We will now consider each of these in turn. 
The kinetic part can be written explicitly as

$$
\mathcal{L}_{V 1}=-\frac{1}{2} \operatorname{str}\left[\left(\partial_{[\mu} A_{\nu]}^{4} T_{4}+\partial_{[\mu} A_{\nu]}^{5} T_{5}+\partial_{[\mu} A_{\nu]}^{6} T_{6}+\partial_{[\mu} A_{\nu]}^{7} T_{7}\right)^{2}\right]
$$

Due to the property of the supertrace of the odd generators given in (5) it is only the cross terms between $T_{4}, T_{5}$ and $T_{6}, T_{7}$ which survive.

$$
\mathcal{L}_{V 1}=-\frac{i}{2}\left(\partial_{[\mu} A_{\nu]}^{4} \partial_{[\mu} A_{\nu]}^{5}+\partial_{[\mu} A_{\nu]}^{6} \partial_{[\mu} A_{\nu]}^{7}\right)=-\frac{1}{4}\left(\partial_{[\mu} A_{\nu]}^{-} \partial_{[\mu} A_{\nu]}^{+}+\partial_{[\mu} B_{\nu]}^{-} \partial_{[\mu} B_{\nu]}^{+}\right),
$$

where we have used the anticommutating properties of the $A_{\mu}^{a}$ 's. In the last step we have replaced the $A_{\mu}^{a}$ by $A_{\mu}^{ \pm}$and $B_{\mu}^{ \pm}$. This kinetic part is reminiscent of the kinetic terms for a charged (i.e. complex) vector field.

Next we work out the form of the interaction terms coming from $\mathcal{L}_{V 2}$. We do this explicitly for $A_{\mu}^{4}$; the results for the other vectors fields can be obtained in a similar manner. The $A_{\mu}^{a}=A_{\mu}^{4}$ part of $\mathcal{L}_{V 2}$ expands like

$$
\mathcal{L}_{V 2}=\frac{g^{2}}{2} \operatorname{str}\left[\left(A_{\mu}^{4} A_{\nu}^{4}\left\{T_{4}, T_{4}\right\}+A_{\mu}^{4} A_{\nu}^{5}\left\{T_{4}, T_{5}\right\}+A_{\mu}^{4} A_{\nu}^{6}\left\{T_{4}, T_{6}\right\}+A_{\mu}^{4} A_{\nu}^{7}\left\{T_{4}, T_{7}\right\}\right)^{2}\right] .
$$

Using the explicit representations of the odd matrices we have $\left\{T_{4}, T_{4}\right\}=\left(T_{3}+T_{8}\right) / 2,\left\{T_{4}, T_{5}\right\}=$ $0,\left\{T_{4}, T_{6}\right\}=T_{1} / 2,\left\{T_{4}, T_{7}\right\}=-T_{2} / 2$. Squaring and using the supertrace results of (4) one finds that (20) becomes

$$
\mathcal{L}_{V 2}=\frac{g^{2}}{16}\left(A_{\mu}^{4} A_{\nu}^{6} A^{4 \mu} A^{6 \nu}+A_{\mu}^{4} A_{\nu}^{7} A^{4 \mu} A^{7 \nu}\right) .
$$

Note that there is no quartic term in $A_{\mu}^{4}$ since the contributions from $T_{3}$ and $T_{8}$ cancel. The contribution from $A_{\mu}^{5}$ looks the same as (21) but with $A_{\mu}^{4} \rightarrow A_{\mu}^{5}$. The $A_{\mu}^{6}$ and $A_{\mu}^{7}$ terms can be obtained by making the exchange $A_{\mu}^{4} \leftrightarrow A_{\mu}^{6}$ and $A_{\mu}^{5} \leftrightarrow A_{\mu}^{7}$. Using the Grassmann character of the $A_{\mu}^{a}$ 's one can see that the $A_{\mu}^{4}$ and $A_{\mu}^{6}$ contributions, and also the $A_{\mu}^{5}$ and $A_{\mu}^{7}$ contributions are the same. In total the interaction part of the vector Lagrangian can be written as

$$
\mathcal{L}_{V 2}=\frac{g^{2}}{16}\left(A_{\mu}^{+} B_{\nu}^{+} A^{-\mu} B^{-\nu}+A_{\mu}^{+} B_{\mu}^{-} A^{-\nu} B^{+\nu}\right) .
$$

In the last line we have written the interaction in terms of $A_{\mu}^{ \pm}, B_{\mu}^{ \pm}$.

The total Lagrangian for the vector Grassmann fields is, $\mathcal{L}_{V 1}+\mathcal{L}_{V 2}$, where $\mathcal{L}_{V 1}$ is a kinetic term and $\mathcal{L}_{V 2}$ gives a nonlinear interaction term between $A_{\mu}^{ \pm}$and $B_{\mu}^{ \pm}$. We assume that the interaction is strong enough that the fields, $A_{\mu}^{ \pm}$and $B_{\mu}^{ \pm}$are permanently confined into condensates

$$
\left\langle A_{\mu}^{+} A^{-\mu}\right\rangle=\left\langle B_{\mu}^{+} B^{-\mu}\right\rangle=v
$$

From the symmetry between the $A_{\mu}^{ \pm}$and $B_{\mu}^{ \pm}$fields we have set their vacuum expectation value to be equal. This conjectured condensation is similar to the gauge variant, mass dimension 2 condensate, in regular Yang-Mills theory, $\left\langle\mathcal{A}_{\mu}^{a} \mathcal{A}^{a \mu}\right\rangle$. Despite being gauge variant this quantity has been shown [22] to have real physical consequences in QCD. Here $\mathcal{A}_{\mu}^{a}$ is a normal $S U(N)$ Yang-Mills field. In [23] a BRST-invariant mass dimension 2 condensate was constructed which was a combination of the quadratic gauge field term $-\left\langle\mathcal{A}_{\mu}^{a} \mathcal{A}^{a \mu}\right\rangle$ - plus a quadratic FadeevPopov [24] ghost field term $-i \alpha\left\langle\mathcal{C}^{a} \overline{\mathcal{C}}^{a}\right\rangle$ - where $\alpha$ was a gauge parameter. In the Landau gauge, $\alpha=0$, this reduced to a pure quadratic gauge field condensate $\left\langle\mathcal{A}_{\mu}^{a} \mathcal{A}^{a \mu}\right\rangle$. Note that the ghost fields, $\mathcal{C}^{a}, \overline{\mathcal{C}}^{a}$, are bosonic, Grassman fields. This mass dimension 2 condensate gives the 
gluon a mass [26]. Estimates have been made for $\sqrt{\left\langle\mathcal{A}_{\mu}^{a} \mathcal{A}^{a \mu}\right\rangle}$ using lattice methods [22, 25], analytical techniques [27] or some mixture. All these methods give a condensate value in the range $\sqrt{\left\langle\mathcal{A}_{\mu}^{a} \mathcal{A}^{a \mu}\right\rangle} \approx 1 \mathrm{GeV}$. From the similarities between the regular gauge field condensate of $[22,23]$ and that on the left hand side of $(23)$ we estimate the vacuum expectation value as $v \approx 1 \mathrm{GeV}^{2}$.

Inserting these vacuum expectation values into (13) yields

$$
\mathcal{L}_{\text {Phantom }}=-\frac{1}{2}\left(\partial_{\mu} \varphi^{8}\right)^{2}-\frac{v}{4} g^{2}\left(\varphi^{8}\right)^{2} .
$$

This is of the form (1) with $V\left(\varphi^{8}\right)=\frac{v}{4} g^{2}\left(\varphi^{8}\right)^{2}$. This will give phantom energy with $w<-1$ if $\frac{g}{2}\left|\varphi^{8}\right| \sqrt{2 v}>\left|\dot{\varphi}^{8}\right|$. If the vacuum expectation value, $v$, changes over time it is possible to cross into (out of) the phantom regime if $v$ increases (decreases). Thus whether one has phantom energy or not would depend on the dynamical evolution of $v$. Such models, where one crosses the "phantom divide", have been considered in [28]. In such models it is usually the sign in front of the kinetic energy term that is modified, whereas in the present case it is a modification of the potential which causes the transition between phantom and non-phantom phases. Further extensions of these "quintom" models can be found in [29].

Inserting the vacuum expectation values into the Lagrangian for the scalar fields $\varphi_{1}, \varphi_{2}, \varphi_{3}$, equation (16) becomes

$$
\mathcal{L}_{D M}=\frac{1}{2}\left(\partial_{\mu} \varphi^{i}\right)^{2}-\frac{v}{4} g^{2}\left(\varphi^{i}\right)^{2} .
$$

The Lagrangian for these fields is for a standard, non-interacting scalar with mass $m=\frac{g}{2} \sqrt{2 v}$. These massive scalar fields could be cold dark matter if $m$ (i.e. $v$ ) is chosen appropriately. For example, using the similarity between the condensate of (23) and the mass dimension condensate of $[22,23]$ one might set $v \approx 1 \mathrm{GeV}^{2}$. This would given $m \approx 1 \mathrm{GeV}$ making $\varphi^{a}$ a viable, cold dark matter candidate.

The original Lagrangian (9) has no coupling to the usual Standard Model fields except through gravity. This would explain why these phantom energy and dark matter fields have not been seen since they could only be detected through their gravitational influence. However if this is the path nature chooses it would be hard, if not impossible, to get any kind of experimental signal of these phantom energy/dark matter candidates. One could introduce some effective coupling between the phantom energy/dark matter fields of (9) and the usual Standard Model fields. More rigorously one might try to use some larger $S U(N / 1)$ group, but with some of the vector fields associated with the even generators and some associated with the odd generators and similarly for the scalar fields. In this way it might be possible to have a new kind of "Grand Unified Theory": from a single Lagrangian one could have Standard Model gauge fields as well as new fields that would be phantom energy and dark matter candidates, instead of extra Grand Unified gauge bosons.

The Grassmann vector fields are an odd feature of this model since they would violate the spin-statistics theorem. These Grassmann vector fields are similar to the Fadeev-Popov ghosts [24]: scalar fields with Fermi-Dirac statistics. The Fadeev-Popov ghosts do not violate the spin-statistics since they never appear as asymptotic states. In order to avoid having the Grassmann vector fields violate the spin-statistics theorem, we have postulated that the composite states, $A_{\mu}^{+} A^{-\mu}$ and $B_{\mu}^{+} B^{-\mu}$ are permanently confined so that the particles associated with $A_{\mu}^{ \pm}$and $B_{\mu}^{ \pm}$never appear as asymptotic states. Since the composites are ordinary fields (integer spin with bosonic statistics) violation of the spin-statistics theorem is avoided. These vectors fields act as a second dark matter component in addition to the three scalar fields $\varphi_{i}$. There have been other recent proposals for dark matter candidates with non-standard relationships between spin and mass dimension. In [30] a spin 1/2 dark matter candidate was proposed 
which has mass dimension 1 . In the present case our vector fields, $A_{\mu}^{ \pm}, B_{\mu}^{ \pm}$, have the same mass dimension (i.e. 1) and statistics (fermionic) as the dark matter candidate in [30], and only differ in the value of spin -1 versus $1 / 2$.

\section{Conclusions}

We have given a model for phantom energy using a modification of the graded Lie algebras models which attempted to give a more unified electroweak theory, or Grand Unified theories. Despite interesting features of the original graded Lie algebra models (e.g. prediction of the Weinberg angle and having both vectors and scalars coming from the same Lagrangian) they had shortcomings. Chief among these was that if one used the correct $S U(N / 1)$ invariant supertrace then some of the vector fields had the wrong sign for the kinetic energy term in the Lagrangian. In the original models the vector fields were associated with the even generators of the algebra and the scalars fields were associated with the odd generators. Here we took the reverse identification (scalar field $\rightarrow$ even generators and vector field $\rightarrow$ odd generators) which led to the wrong sign kinetic energy term coming from a scalar field rather than from a vector field. The wrong sign scalar field, $\varphi^{8}$, gives a model of phantom energy, while the other scalar fields, $\varphi^{i}$, and the vector fields, $A_{\mu}^{a}$, act as dark matter components. In the way our model is formulated here all the fields are truly dark in that they have no coupling to any of the Standard Model fields and would thus only be detectable via their gravitational interaction. This would make the experimental detection of these dark fields impossible through non-gravitational interactions. However the above is intended only as a toy model of how a phantom energy field can emerge naturally from a gauge theory with a graded Lie algebra. A more experimentally testable variation of the above toy model could have some coupling between the scalar and vector fields of the present model and the Standard Model fields. Such a coupling could be introduced in a phenomenological fashion via some ad hoc coupling. A more interesting option would be to consider some larger graded algebra, such as $S U(N / 1)$. Some of the fields could be given the standard assignment of even or odd generators (i.e. as in (6)) while others could be given the assignment in (8). The fields given the standard assignment would give standard gauge fields, while fields given the non-standard assignment would give phantom energy and dark matter fields. This would give a new type of "Grand Unified Theory" with the phantom energy and dark matter fields replacing the extra gauge bosons of ordinary Grand Unified Theories. Other authors [31] have used non-standard gauge groups such as $S O(1,1)$ to give models of phantom energy.

An important feature of the above model is the assumption that the Grassmann vector fields form permanently confined condensates. This was a crucial to our phantom energy model since it leads to a condensate of the $A_{\mu}^{ \pm}$and $B_{\mu}^{ \pm}$fields. This in turn gave a potential $V\left(\varphi^{8}\right)=\frac{v}{4} g^{2}\left(\varphi^{8}\right)^{2}$ for the $\varphi^{8}$ field which was of the correct form to allow $\varphi^{8}$ to act as phantom energy. Aside from the present application to phantom energy one might try to use the above mechanism to generate standard symmetry break by starting with a graded Lie algebra but using all vector fields rather than mixing vector and scalar. In this way some of the vector fields would be standard vector fields, while other would be Grassmann vector fields. By the above mechanism the Grassmann vector fields would form condensates which would then give masses to the standard vector fields i.e. one would have a Higgs mechanism with only vector fields).

An additional avenue for future investigation is to see if one could have a phantom energy model with the original graded Lie algebra models (i.e. with vector fields assigned to even generators and scalars to odd) but using the supertrace. One would then have the problem of some of the vector fields having the wrong sign in the kinetic term, but this might then give a phantom energy model with a vector rather than scalar field.

As a final note the dark energy fields (those connected with the even generators) considered here violate all the known energy conditions which are normally required of quantum fields. 
The reason for taking this drastic step is that it gives a model for phantom dark energy which is thought to drive the observed expansion of the Universe. If it turns out that the indications for $w<-1$ are not correct (i.e. if $-1<w<-1 / 3$ ) then there would be no need for phantom dark energy; "ordinary" dark energy would do. Recently, [32] there has been a proposal that dark energy effects can be entirely explained by non-localized gravitational energy or rather gradients in gravitational energy. If this proposal is correct then there would be no need that we can see for dark energy in any form - phantom or otherwise.

\section{Acknowledgments}

DS acknowledges the CSU Fresno College of Science and Mathematics for a sabbatical leave during the period when this work was completed, and a CSM 2007 Professional Development Grant to attend Symmetry-200\%.

\section{References}

[1] Dondi P.H., Jarvis P.D., A supersymmetric Weinberg-Salam model, Phys. Lett. B 84 (1979), 75-78, Erratum, Phys. Lett. B 87 (1979), 403-406.

Ne'eman Y., Irreducible gauge theory of a consolidated Weinberg-Salam model, Phys. Lett. B 81 (1979), 190-194.

Fairlie D.B., Higgs' fields and the determination of the Weinberg angle, Phys. Lett. B 82 (1979), 97-100.

Squires E.J., On a derivation of the Weinberg-Salam model, Phys. Lett. B 82 (1979), 395-397.

Taylor J.G., Electroweak theory in $S U(2 / 1)$, Phys. Lett B 83 (1979), 331-334.

[2] Taylor J.G., Superunification in SU(5/1), Phys. Rev. Lett. 43 (1979), 824-826.

[3] Eccelstone R.E., A critique of supersymmetric Weinberg-Salam models, J. Phys. A: Math. Gen. 13 (1980), 1395-1408.

Eccelstone R.E., The Weinberg angle in the supersymmetric Weinberg-Salam model, Phys. Lett. B 116 (1982), 21-22.

[4] Caldwell R.R., A phantom menace?, Phys. Lett. B 545 (2002), 23-29, astro-ph/9908168.

Caldwell R.R., Kamionkowski M., Weinberg N.N., Phantom energy and cosmic doomsday, Phys. Rev. Lett. 91 (2003), 071301, 4 pages, astro-ph/0302506.

[5] Carroll S.M., Hoffman M., Trodden M., Can the dark energy equation-of-state parameter $w$ be less than -1 ?, Phys. Rev. D 68 (2003), 023509, 11 pages, astro-ph/0301273.

[6] Riess A.G. et al., Observational evidence from supernovae for an accelerating universe and a cosmological constant, Astronom. J. 116 (1998), 1009-1038, astro-ph/9805201.

Riess A.G. et al., The case for an accelerating universe from supernovae, Publ. Astronom. Soc. Pacific 112 (2000), 1284-1299, astro-ph/0005229.

[7] Perlmutter S. et al., Measurements of $\Omega$ and $\Lambda$ from 42 high redshift supernovae, Astrophys. J. 517 (1999), 565-586, astro-ph/9812133.

[8] Zlatev I., Wang L., Steinhart P.J., Quintessence, cosmic coincidence, and the cosmological constant, Phys. Rev. Lett. 82 (1999), 896-899, astro-ph/9807002.

[9] Deffayet C., Dvali G., Gabadadze G., Accelerated universe from gravity leaking to extra dimensions, Phys. Rev. D 65 (2002), 044023, 9 pages, astro-ph/0105068.

[10] Deffayet C., Landau S.J., Raux J., Zaldarriaga M., Astier P., Supernovae, CMB, and gravitational leakage into extra dimensions, Phys. Rev. D 66 (2002), 024019, 10 pages, astro-ph/0201164.

[11] Kamenshchik A., Moschella U., Pasquier V., An alternative to quintessence, Phys. Lett. B 511 (2001), 265-268, gr-qc/0103004.

[12] Gonzalez-Diaz P.F., k-essential phantom energy: doomsday around the corner?, Phys. Lett. B 586 (2004), 1-4, astro-ph/0312579.

[13] Gonzalez-Diaz P.F., Axion phantom energy, Phys. Rev. D 69 (2004), 063522, 6 pages, hep-th/0401082.

[14] Sahni V., Dark matter and dark energy, Lect. Notes Phys. 653 (2004), 141-180, astro-ph/0403324. 
[15] Bronnikov K., Scalar-tensor theory and scalar charge, Acta. Phys. Pol. B 4 (1973), 251-266.

Ellis H., Ether flow through a drainhole - a particle model in general relativity, J. Math. Phys. 14 (1973), 104-118.

[16] Kodama T., General relativistic nonlinear field: a kink solution in a generalized geometry, Phys. Rev. D 18 (1978), 3529-3534.

Armendáriz-Picón C., On a class of stable, traversable Lorentzian wormholes in classical general relativity, Phys. Rev. D 65 (2002), 104010, 10 pages, gr-qc/0201027.

Lobo F.S.N., Phantom energy traversable wormholes, Phys. Rev. D 71 (2005), 084011, 8 pages, gr-qc/0502099.

Sushkov S.V., Wormholes supported by a phantom energy, Phys. Rev. D 71 (2005), 043520, 5 pages, gr-qc/0502084.

[17] Cline J., Jeon S., Moore G., The phantom menaced: constraints on low-energy effective ghosts, Phys. Rev. D 70 (2004), 043543, 4 pages, hep-ph/0311312.

[18] Hannestad S., Mortsell E., Probing the dark side: constraints on the dark energy equation of state from CMB, large scale structure and type Ia supernovae, Phys. Rev. D 66 (2002), 063508, 5 pages, astro-ph/0205096.

Melchiori A. et al., The state of the dark energy equation of state, Phys. Rev. D 68 (2003), 043509, 7 pages, astro-ph/0211522.

Knop R.A. et al., New constraints on $\Omega_{\mathrm{M}}, \Omega_{\Lambda}$, and $w$ from an independent set of eleven high-redshift supernovae observed with HST, Astrophys. J. 598 (2003), 102-137, astro-ph/0309368.

Spergel D.N. et al., First year Wilkinson microwave anisotropy probe (WMAP) observations: determination of cosmological parameters, Astrophys. J. Suppl. 148 (2003), 175-194, astro-ph/0302209.

Tegmark M. et al., Cosmological parameters from SDSS and WMAP, Phys. Rev. D 69 (2004), 103501, 26 pages, astro-ph/0310723.

[19] Jassal H., Bagla J., Padmanabhan T., Observational constraints on low redshift evolution of dark energy: How consistent are different observations?, Phys. Rev. D 72 (2005), 103503, 21 pages, astro-ph/0506748.

[20] Chaves M., Singleton D., Phantom energy from graded algebras, Modern Phys. Lett. A 22 (2007), 29-40, hep-th/0603160.

[21] Cai R., Wang A., Cosmology with interaction between phantom dark energy and dark matter and the coincidence problem, J. Cosmol. Astropart. Phys. 2005 (2005), no. 3, 002, 17 pages, hep-th/0411025.

[22] Boucaud Ph. et al., Consistent OPE description of gluon two point and three point Green function?, Phys. Lett. B 493 (2000), 315-324, hep-ph/0008043.

Boucaud $\mathrm{Ph}$. et al., Testing Landau gauge OPE on the lattice with a $\left\langle A^{2}\right\rangle$ condensate, Phys. Rev. D 63 (2001), 114003, 9 pages, hep-ph/0101302.

Gubarev F.V., Stodolsky L., Zakharov V.I., On the significance of the vector potential squared, Phys. Rev. Lett. 86 (2001), 2220-2222, hep-ph/0010057.

Gubarev F.V., Zakharov V.I., On the emerging phenomenology of $\left\langle\left(A_{\mu}^{a}\right)_{\min }^{2}\right\rangle$, Phys. Lett. B 501 (2001), 28-36, hep-ph/0010096.

[23] Kondo K.-I., Vacuum condensate of mass dimension 2 as the origin of mass gap and quark confinement, Phys. Lett. B 514 (2001), 335-345, hep-th/0105299.

[24] Faddeev L.D., Popov V.N., Feynman diagrams for the Yang-Mills field, Phys. Lett. B 25 (1967), 29-30.

[25] Li X., Shakin C.M., Description of gluon propagation in the presence of an $A^{2}$ condensate, Phys. Rev. D 71 (2005), 074007, 13 pages, hep-ph/0410404.

[26] Dzhunushaliev V.D., Singleton D., Ginzburg-Landau equation from SU(2) gauge field theory, Modern Phys. Lett. A 18 (2003), 955-966, hep-th/0210287.

Dzhunushaliev V.D., Singleton D., Effective 't Hooft-Polyakov monopoles from pure $S U(3)$ gauge theory, Modern Phys. Lett. A 18 (2003), 2873-2886, hep-ph/0306202.

[27] Dudal D. et al., A determination of $A^{2}(\mu)$ and the nonperturbative vacuum energy of Yang-Mills theory in the Landau gauge, Phys. Lett. B 562 (2003), 87-96, hep-th/0302128.

[28] Feng B., Wang X., Zhang X., Dark energy constraints from the cosmic age and supernova, Phys. Lett. B 607 (2005), 35-41, astro-ph/0404224.

Vikman A., Can dark energy evolve to the phantom?, Phys. Rev. D 71 (2005), 023515, 14 pages, astro-ph/0407107.

Andrianov A., Cannata F., Kamenshchik A., Smooth dynamical crossing of the phantom divide line of a scalar field in simple cosmological models, Phys. Rev. D $\mathbf{7 2}$ (2005), 043531, 8 pages, gr-qc/0505087.

Nojiri S., Odintsov S., Inhomogeneous equation of state of the universe: phantom era, future singularity, and crossing the phantom barrier, Phys. Rev. D 72 (2005), 023003, 12 pages, hep-th/0505215. 
[29] Feng B. et al., Oscillating quintom and the recurrent universe, Phys. Lett. B 634 (2006), 101-105, astro-ph/0407432.

Xia J., Feng B., Zhang X., Constraints on oscillating quintom from supernova, microwave background and galaxy clustering, Modern Phys. Lett. A 20 (2005), 2409-2416, astro-ph/0411501.

Li M., Feng B., Zhang X., A Single scalar field model of dark energy with equation of state crossing -1 , J. Cosmol. Astropart. Phys. 2005 (2005), no. 12, 002, 8 pages, hep-ph/0503268.

Zhao G. et al., Perturbations of the quintom models of dark energy and the effects on observations, Phys. Rev. D 72 (2005), 123515, 16 pages, astro-ph/0507482.

Xia J. et al., Observing dark energy dynamics with supernova, microwave background and galaxy clustering, Phys. Rev. D 73 (2006), 063521, 8 pages, astro-ph/0511625.

[30] Ahluwalia-Khalilova D.V., Grumiller D., Dark matter: a spin one half fermion field with mass dimension one?, Phys. Rev. D $\mathbf{7 2}$ (2005), 067701, 4 pages, hep-th/0410192.

Ahluwalia-Khalilova D.V., Grumiller D., Spin half fermions with mass dimension one: theory, phenomenology, and dark matter, J. Cosmol. Astropart. Phys. 2005 (2005), no. 7, 012, 72 pages, hep-th/0412080.

[31] Wei Y., Big rip in $S O(1,1)$ phantom universe, gr-qc/0502077.

[32] Wiltshire D., Exact solution to the averaging problem in cosmology, Phys. Rev. Lett. 99 (2007), 251101, 4 pages, arXiv:0709.0732.

Wiltshire D., Cosmic clocks, cosmic variance and cosmic averages, New J. Phys. 9 (2007), 377, 66 pages, gr-qc/0702082.

Wiltshire D., Gravitational energy and cosmic acceleration, arXiv:0712.3982. 